\title{
Incidencia De Los Aspectos Psicosociales En La Deserción De Los Estudiantes Universitarios
}

\author{
María Guadalupe Escobar Murillo \\ Magister en Docencia Universitaria y Administración Educativa \\ Docente de la Escuela Superior Politécnica de Chimborazo, \\ Centro de Idiomas, Ecuador \\ Arellano Díaz Mario Alfonso \\ Master en Dirección De Empresas \\ Docente de la Escuela Superior Politécnica de Chimborazo, \\ Facultad de Administración de Empresas, Ecuador \\ Rodríguez Pillajo Lucio Armando \\ Magister en Docencia Universitaria \\ Docente de la Escuela Superior Politécnica de Chimborazo, \\ Centro de Idiomas, Ecuador \\ Nanci Margarita Inca Chunata \\ Magister en Docencia Universitaria y Administración Educativa \\ Docente de la Escuela Superior Politécnica de Chimborazo, \\ Centro de Idiomas, Ecuador
}

Doi:10.19044/esj.2018.v14n19p206 URL:http://dx.doi.org/10.19044/esj.2018.v14n19p206

\begin{abstract}
This paper focuses on determining the incidence of the psychosocial aspects of the dropout of students from the School of Marketing at the School of Business Administration of the Higher Polytechnic School in Chimborazo. In this study, the dependent variable include Psychological, Socioeconomic aspects, and its environment. In the dependent variable, the problems of the students, higher education, and social problems were considered. The research was a bibliographic and field research. It was, therefore, developed within the critical and qualitative paradigms. This is attributed to the fact that a dynamic reality was assumed for the diagnosis, analysis, and determination of results. For the collection of data, a survey validated by academic peers was used and executed with a data collection plan for 186 students from first to sixth level (27 teachers and 2 authorities). The data obtained was submitted to a data processing plan, and the analysis and interpretation of the data was later performed. Finally, each psychosocial aspect was submitted to a statistical test which shows that the university desertion has multi-causal origin. It also
\end{abstract}


highlights that the most recurrent have to do with the psychosocial factors that affects the students.

Keywords: Incidence, Psychosocial aspects, University desertion, Students

\section{Resumen}

El objetivo de la presente investigación, fue determinar la incidencia de los aspectos psicosociales en la deserción de los estudiantes de la Escuela de Marketing de la Facultad de Administración de Empresas de la Escuela Superior Politécnica de Chimborazo; para ello, se dimensionaron la variable dependiente en aspectos Psicológicos, socioeconómicos y su entorno; mientras que en la variable dependiente se consideró los problemas de los estudiantes, de la educación superior y los problemas sociales. La investigación fue bibliográfica y de campo. Se desarrolló dentro de los paradigmas crítico y cualitativo; ya que se asumió una realidad dinámica para el diagnosticó, análisis y determinación de resultados. Para la recolección de la información se utilizó una encuesta validada por pares académicos y ejecutada con un plan de recolección de información a 186 estudiantes de primero a sexto nivel, 27 docentes y 2 autoridades. La información obtenida fue sometida a un plan de procesamiento de información, para posterior realizar el análisis, interpretación y finalmente someter cada aspecto psicosocial a una prueba estadística; que permitió determinar que la deserción universitaria tiene origen multicausal y que las más recurrentes tienen que ver con los factores psicosociales que afectan a los estudiantes.

Palabras Claves: Incidencia, Aspectos psicosociales, Deserción Universitaria, Estudiantes

\section{INTRODUCCIÓN}

Según la Organización de Estados Iberoamericanos para la Educación (OEI), en su revista Iberoamericana de Educación $\mathrm{N}^{\circ} 50$ (2009). El siglo XX ha sido el siglo de la democratización política de las sociedades latinoamericanas y derivado de ello, del inicio del acceso masivo a los diversos servicios sociales públicos. En el campo educativo esta evolución comenzó con la masificación de la educación básica; pasó luego a expresarse en la expansión de la educación media y a derivarse en crecientes tasas de aumento de la cobertura de la educación superior hacia su universalización, que se constituye como una de las tendencias más fuertes desde las últimas décadas. Según el estudio este proceso continuará durante un buen trecho del siglo XXI, con un creciente corrimiento hacia la educación permanente y estudios de posgrado en todos sus niveles. "Esta masificación del acceso a la educación y la formación de capital humano están produciendo tanto una democratización 
de la propia educación como una transformación en la conformación de las elites sociales" (OEI, $\left.2009 \mathrm{~N}^{\circ} 50\right)$. Es este un proceso a escala global que en Estados Unidos se dinamiza desde los años cincuenta; en Europa, desde los años sesenta; en Asia, desde la década de 1970, y en América Latina, desde la siguiente. Tal dinámica de universitarización de la educación concluyó en los países centrales al alcanzarse los niveles de universalización de la cobertura, en tanto que en nuestra región dicha dinámica aún está en proceso de desarrollo y consolidación, a través de etapas que van marcando las características de las fases educativas asociadas al ingreso de nuevos tipos de estudiantes.

Las afectaciones psicosociales están relacionadas con las características personales de los estudiantes, que constituyen una de las causas importantes que generan una alta desmotivación para la deserción universitaria y están relacionadas a la ausencia y claridad de manejar un proyecto de vida, que generalmente están asociado al poco compromiso que tienen con sus metas, y a una pobre conducta de logro. Tener poca fortaleza personal, aporta a esta causa; pero sobre todo: la poca capacidad de ajuste saludable a la adversidad, que es una de las principales causas que generan frecuentes momentos de desmotivación que sumados a otros factores, confluyen a estar expuestos a la Deserción universitaria.

Teresita Selame (1994), anota en su estudio sociológico los factores que inciden en la deserción estudiantil como "el abandono, voluntario o forzado, de la carrera en la que estos se matricularon. Entendiendo por razones forzadas al alumno que es eliminado o abandona sus estudios por motivos académicos y voluntario o propiamente desertor al alumno que toma por sí mismo la decisión de dejar la carrera" (Álvarez, 1997).

"La deserción se puede explicar cómo el resultado de distintos vectores que afectan al estudiante. Por ello, es necesario estudiar y analizar directamente al desertor, ya que la deserción es un fenómeno inherente a la vida estudiantil, relacionado a procesos dinámicos de decisión, rendimiento académico y de la eficiencia del sistema educativo en general" (Díaz, 2009).

Cuando se analiza la deserción estudiantil se deben considerar también aspectos relacionados con: "el auto-concepto que surge de la interrelación de tres instancias: autoimagen (visión que la persona tiene de sí en un momento particular), imagen social (lo que la persona cree que los demás piensan de ella) e imagen ideal (cómo le gustaría ser)" (Montero, 2000).

"Los modelos sociológicos enfatizan respecto a la influencia en la deserción de factores externos al individuo, adicionales a los psicológicos" Spady (1970), citado por (Diaz, 2008).

La deserción es el resultado de la falta de integración de los estudiantes en el entorno de la educación superior; se menciona que el medio familiar es una de las muchas fuentes que expone a los estudiantes a 
influencias, expectativas y demandas, las que a su vez afectan su nivel de integración social en la universidad; por otro lado la congruencia y posibilidades de la Organización actúa directamente sobre el rendimiento académico, el desarrollo intelectual, el apoyo de pares y la integración social.

Según Cortéz (2005), en su estudio sobre repitencia y deserción en la educación superior en Bolivia, manifiesta que la deserción en las universidades públicas de Bolivia tienen niveles elevados; en el caso de las carreras seleccionadas para el estudio: la deserción especifica alcanza al $50 \mathrm{y}$ $60 \%$. Proporcionalmente las tasas de Titulación son bajas, situándose entre el 19 y $27 \%$.

Otra de las causas mediatas que inciden en la deserción universitaria, es la influencia del entorno social y dentro de este, se puede identificar como causas la influencia del entorno familiar relacionado tanto con la etapa de escogimiento de una carrera universitaria, como por otros elementos de este mismo entorno que los afectan, así por ejemplo: el estrato social al que pertenecen, la educación de los padres, la desestructuración familiar y otras. Del mismo modo el entorno universitario y particularmente la relación profesor alumno, viciada a veces de maltrato de parte del profesor tiene su nivel de incidencia en la deserción.

Las dificultades que los estudiantes tienen para financiar su carrera universitaria generalmente asociados a los gastos de colegiatura pero sobre todo a los gastos para su sustento (alimentación, vivienda, material educativo, transporte y otros) son factores socioeconómicos considerados causales de deserción universitaria afectados ya sea por las propias condiciones económicas "limitadas" de los estudiantes así como por las dificultades de acceso a opciones de financiamiento para sus estudios; se entiende que al término de este estudio (año 2016) las condiciones de acceso a estos mecanismos de financiamiento para estudios a través del Instituto Ecuatoriano de Crédito Educativo y Becas han mejorado y facilitan su acceso.

Según el estudio "Ecuador Desarrollo y la Nueva Generación", realizado por el Banco Mundial, que analiza la situación actual de las juventudes del país concluye: que los hogares empobrecidos aumentaron los índices de deserción del sistema educativo y temprana incorporación al mercado laboral mayormente informal. A partir de los 20 años existe una alta propensión a abandonar los estudios superiores; la presión laboral, los recursos financieros para el estudio, así como el tiempo se reduce con la edad. La deserción es superior en las áreas rurales. Entre los datos el estudio revela que pese al incremento en el acceso a educación, el 26\% de la población joven no ha terminado la educación primaria, el 69\% la educación secundaria y apenas 8 de cada cien jóvenes que ingresan a la universidad obtienen un título universitario. 
Según González (2005), la deserción tiene consecuencias sociales en términos de las expectativas de los estudiantes y sus familias; emocionales por la disonancia entre las aspiraciones de los jóvenes y sus logros y, también, importantes consecuencias económicas tanto para las personas como para el sistema en su conjunto.

La deserción universitaria, por sus dimensiones, se ha convertido en un fenómeno muy común en la mayoría de los países latinoamericanos, razón por lo cual se viene planteando como uno de los problemas prioritarios a ser investigado y adecuadamente tratado. En ese sentido, el Instituto Internacional de la UNESCO para la Educación Superior en América Latina y el Caribe (IESALC) plantea la necesidad de realizar estudios en toda la región, que ofrezcan luz sobre los factores causales y la búsqueda de solución al problema. (Brea, 2005).

Para apoyar este estudio es importante conjugar las definiciones de deserción dadas por Tinto (1982) y Giovagnoli (2002); se puede definir entonces a la deserción como una situación a la que se enfrenta un estudiante cuando aspira y no logra concluir su proyecto educativo, considerándose como desertor a aquel individuo que siendo estudiante de una institución de educación superior no presenta actividad académica durante dos semestres académicos consecutivos lo cual equivale a un año de inactividad académica.

La Escuela de Marketing de la facultad de Administración de Empresas en los últimos años ha evidenciado una disminución en los egresados frente a la demanda de matrícula; según datos de la Secretaría de la Escuela de Marketing, el 2009 de 50 alumnos y alumnas matriculados/as en el primer semestre aprueban al semestre inmediato superior 10 alumnos/as, 40 estudiantes no aprueban por diferentes causas, y este comportamiento se observa en los subsiguientes niveles. Problemática que repercute notablemente en este centro de estudios con el consiguiente malgasto de recursos económicos, materiales, talento humano y lo que es más el hecho de que muchos de los estudiantes no terminen una carrera universitaria.

El estudio de la deserción de la educación superior es complejo, ya que implica no solo una variedad de perspectivas, sino también una gama de diferentes tipos de abandono. Tinto (1989), sin embargo con la presente investigación se pretendió acercarse más a las causas de carácter psicosocial que inciden en el abandono de la carrera universitaria.

\section{METODOLOGÍA}

La investigación se desarrolló dentro del paradigma crítico propositivo, ya que se diagnosticó y analizó la situación de la problemática. Se enmarcó dentro del paradigma cualitativo, ya que se comparó fenómenos sociales con un enfoque contextualizado asumiendo una realidad dinámica. 
La presente investigación es documental, pues se basó en estudios de autores que han realizado investigaciones respecto a factores psicosociales de estudiantes universitarios, así como estudios sobre deserción universitaria. Tiene el propósito de detectar, ampliar y profundizar diferentes enfoques teóricos, conceptualizaciones de diversos criterios de diversos autores sobre el tema que se investiga.

La investigación fue de campo, ya que permitió testificar la problemática con una muestra igual a la población de 186 estudiantes entre los 19 y los 22 años de edad, 2 Autoridades y 10 Docentes universitarios de la Escuela de Marketing de la Facultad de Administración de Empresas de la Escuela Superior Politécnica de Chimborazo de Riobamba.

Tabla 1. Población

\begin{tabular}{|l|c|}
\hline Autoridades & 2 \\
Docentes & 10 \\
Estudiantes & 186 \\
\hline
\end{tabular}

Elaborado por: Autores.

Como la población estudiantil es superior a 150 personas, se determina una muestra de acuerdo a la siguiente fórmula:

$\begin{aligned} & \mathrm{n}= \mathrm{Z}^{2} \mathrm{PQN} \\ & \mathrm{Z}^{2} \mathrm{PQ}+\mathrm{Ne}^{2}\end{aligned}$

$\mathrm{n}=$ Tamaño de la muestra

$\mathrm{Z}=$ Nivel de confiabilidad $95 \% \mathrm{Z}=1.96$

$\mathrm{P}=$ Probabilidad de ocurrencia 0.35

$\mathrm{Q}=$ Probabilidad de no ocurrencia $1-0.35=0.65$

$\mathrm{N}=$ Población 215

$\mathrm{E}=$ Error de muestreo 0.05

Tabla 2. Muestra: Nivel de confiabilidad del $95 \%$

\begin{tabular}{|c|c|}
\hline Informantes & $\mathbf{N}^{\circ}$ \\
\hline Autoridades & 2 \\
Docentes & 10 \\
Estudiantes & 121 \\
\hline Total & 133 \\
\hline
\end{tabular}

Elaborado por: Autores.

En la investigación, se incluyó las variables con hipótesis a fin de medir el grado de relación, llegando al nivel explicativo con la finalidad de detectar factores determinantes de ciertos comportamientos evidenciados en la muestra; así como llegar a la comprobación de hipótesis con la finalidad de aproximarse a las causas del problema estudiado.

La investigación es descriptiva, ya que se determinó como es y cómo está la situación de las variables, teniendo como objeto el obtener el 
conocimiento de los rangos extremos del fenómeno, es decir de aquello que se investiga.

Se utilizó la técnica de encuesta y entrevista, a la cual los informantes responden por escrito a preguntas sometidas a pares académicos previamente entregadas. Las preguntas son de diversa naturaleza: abiertas o de opinión y cerradas. El test fue aplicado a los integrantes de la muestra, que permitió conocer la percepción sobre los aspectos psicosociales y la deserción.

Aplicado los instrumentos, se establecieron los resultados, se codificaron, tabularon y elaboraron los cuadros de distribución, utilizando la técnica de porcentajes.

Mediante Microsoft Excel, se realizó la representación de los datos con estadística descriptiva y se utilizó frecuencias, gráficos, y porcentajes; de tal forma, que se pueda visualizar claramente los resultados de cada una de las respuestas dadas por los participantes sobre los datos generales, elementos, y aspectos psicosociales en la deserción de los estudiantes.

Se aplicó la encuesta a la muestra, a fin de analizar las principales razones que llevan a los estudiantes a abandonar los estudios universitarios. La segunda fase consistió en analizar los resultados, para identificar los factores psicosociales que afectan a los estudiantes en el transcurso de su vida universitaria y aportar a la búsqueda de alternativas de solución al problema de la deserción universitaria.

Para llegar a la meta, se identificó los aspectos psicosociales de los estudiantes que representan la muestra de la investigación; luego se analizó la intención de deserción en los estudiantes en relación a la elección de la carrera; además se consideró la relación entre la deserción y motivación para estudiar. Los datos obtenidos se sometieron a una prueba estadística, que permitió la comprobación de las hipótesis y la determinación de las conclusiones.

\section{RESULTADOS}

\section{Deserción y elección de la carrera}

\begin{tabular}{|c|c|c|c|c|c|c|}
\hline & \multicolumn{4}{|c|}{ ¿Por qué eligió su carrera? } & \multirow[b]{2}{*}{ Total } \\
\hline & & Vocación & $\begin{array}{c}\text { Influencia } \\
\text { familiar }\end{array}$ & Amigos & $\begin{array}{c}\text { Otros } \\
\text { factores }\end{array}$ & \\
\hline \multirow{4}{*}{$\begin{array}{l}\text { ¿Quisiera o ha } \\
\text { querido retirarse } \\
\text { de la carrera? }\end{array}$} & $\mathbf{S i}$ & 4 & 2 & 0 & 4 & 10 \\
\hline & No & 50 & 8 & 2 & 23 & 83 \\
\hline & A veces & 12 & 4 & 3 & 6 & 25 \\
\hline & Total & 66 & 14 & 5 & 33 & 118 \\
\hline
\end{tabular}

Fuente: Investigación de campo

Elaboración: Autores 


\section{Planteamiento de hipótesis}

Ho: El deseo de retirarse de la carrera y el motivo elección de la misma son independientes.

H1: El deseo de retirarse de la carrera y el motivo elección de la misma son dependientes.

Establecimiento del nivel de significancia

$$
\alpha=0,05
$$

\section{Cálculo del estadístico de prueba}

Tabla 4. Estadístico de prueba 1

\begin{tabular}{|c|c|c|}
\hline $\boldsymbol{f o}$ & $\boldsymbol{f e}$ & $\frac{(\boldsymbol{f o}-\boldsymbol{f e})^{\mathbf{2}}}{\boldsymbol{f e}}$ \\
\hline 4 & 5,593 & 0,454 \\
\hline 2 & 1,186 & 0,559 \\
\hline 0 & 0,424 & 0,424 \\
\hline 4 & 2,797 & 0,517 \\
\hline 50 & 46,424 & 0,275 \\
\hline 8 & 9,847 & 0,346 \\
\hline 2 & 3,517 & 0,654 \\
\hline 23 & 23,212 & 0,002 \\
\hline 12 & 13,983 & 0,281 \\
\hline 4 & 2,966 & 0,360 \\
\hline 3 & 1,059 & 3,558 \\
\hline 6 & 6,992 & 0,141 \\
\hline \multicolumn{2}{|r|}{ suma } & $\mathbf{7 , 5 6 9}$ \\
\cline { 3 - 3 }
\end{tabular}

Fuente: Investigación de campo

Elaboración: Autores

Tabla 5. Pruebas chi-cuadrado 1

\begin{tabular}{|l|c|c|c|} 
& Valor & Gl & Valor $\mathrm{p}$ \\
\hline Chi-cuadrado de Pearson & 7,569 & 6 & 0,271 \\
Razón de verosimilitudes & 6,82 & 6 & 0,338 \\
$\mathrm{~N}^{\circ}$ de casos válidos & 118 & & \\
\hline
\end{tabular}

Fuente: Investigación de campo

Elaboración: Autores 
Cálculo del valor crítico y zona de rechazo

\begin{tabular}{|c|c|}
\hline $\mathrm{f}=$ & 3 \\
\hline $\mathrm{c}=$ & 4 \\
\hline $\mathrm{gl}=$ & 6 \\
\hline Valor crítico & $\mathbf{1 2 , 5 9 2}$ \\
\hline
\end{tabular}

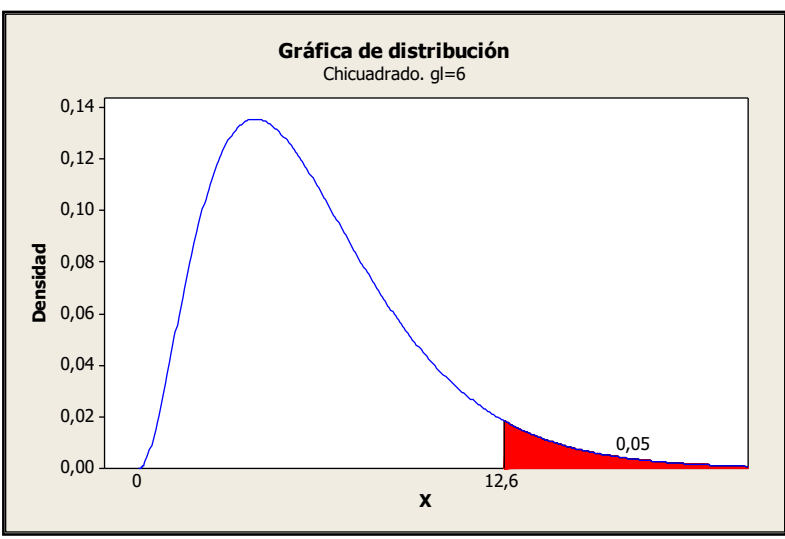

Grafico 1. Valor crítico y zona de rechazo 1

\section{Regla de decisión}

Rechazar Ho si el estadístico de prueba es mayor que el valor crítico

No rechazar Ho si el estadístico de prueba es menor que el valor crítico

\section{Conclusión}

Como el estadístico de prueba es menor que el valor crítico no se puede rechazar Ho, entonces, el deseo de retirarse de la carrera y el motivo de elección de la misma son independientes

\section{Deserción y motivación para estudiar}

\begin{tabular}{|c|c|c|c|c|c|c|}
\hline & \multicolumn{4}{|c|}{ Tu motivación para estudiar es } & \multirow[b]{2}{*}{ Total } \\
\hline & & Alta & Media & Baja & Muy baja & \\
\hline \multirow{4}{*}{$\begin{array}{c}\text { ¿Quisiera o } \\
\text { ha querido } \\
\text { retirarse de la } \\
\text { carrera? }\end{array}$} & $\mathbf{S i}$ & 6 & 3 & 1 & 0 & 10 \\
\hline & No & 52 & 31 & 0 & 1 & 84 \\
\hline & A veces & 12 & 12 & 0 & 0 & 24 \\
\hline & Total & 70 & 46 & 1 & 1 & 118 \\
\hline
\end{tabular}

Elaboración: Autores

\section{Planteamiento de hipótesis}

Ho: El deseo de retirarse de la carrera y la motivación para estudiar son independientes.

H1: El deseo de retirarse de la carrera y la motivación para estudiar son dependientes. 
Establecimiento del nivel de significancia

$\alpha=0,05$

\section{Estadístico de prueba}

Tabla 7. Pruebas de chi-cuadrado 2

\begin{tabular}{|l|c|c|c|}
\hline & Valor & Gl & Valor p \\
\hline Chi-cuadrado de Pearson & 12,699 & 6 & 0,048 \\
Razón de verosimilitudes & 7,087 & 6 & 0,313 \\
N de casos válidos & 118 & & \\
\hline
\end{tabular}

Fuente: Investigación de campo

Elaboración: Autores

Cálculo del valor crítico y zona de rechazo

\begin{tabular}{|c|c|}
\hline $\mathrm{f}=$ & 3 \\
\hline $\mathrm{c}=$ & 4 \\
\hline $\mathrm{gl}=$ & 6 \\
\hline Valor crítico & $\mathbf{1 2 , 5 9 2}$ \\
\hline
\end{tabular}

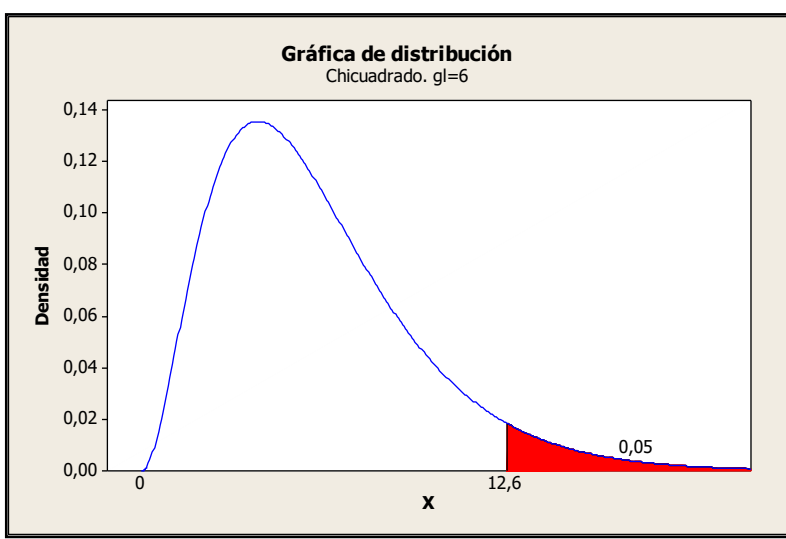

Gráfico No. 2 Valor crítico y zona de rechazo 2

\section{Regla de decisión}

Rechazar Ho si el estadístico de prueba es mayor que el valor crítico

No rechazar Ho si el estadístico de prueba es menor que el valor crítico

\section{Conclusión}

Como el estadístico de prueba es mayor que el valor crítico se procede a rechazar Ho, entonces, el deseo de retirarse de la carrera y la motivación para estudiar son dependientes. 


\section{Deserción y dificultades para estudiar}

Tabla 8. Dificultades para estudiar

\begin{tabular}{|c|c|c|c|c|c|}
\hline & & \multicolumn{3}{|c|}{ ¿Tiene dificultades para estudiar? } & \multirow[b]{2}{*}{ Total } \\
\hline & & $\mathbf{S i}$ & No & A veces & \\
\hline \multirow{3}{*}{$\begin{array}{l}\text { ¿Quisiera o ha querido } \\
\text { retirarse de la carrera? }\end{array}$} & $\mathbf{S i}$ & 3 & 0 & 7 & 10 \\
\hline & No & 3 & 31 & 50 & 84 \\
\hline & A veces & 3 & 5 & 17 & 25 \\
\hline & Total & 9 & 36 & 74 & 119 \\
\hline
\end{tabular}

Fuente: Investigación de campo

Elaboración: Autores

\section{Planteamiento de hipótesis}

Ho: El deseo de retirarse de la carrera y la dificultad para estudiar son independientes.

H1: El deseo de retirarse de la carrera y dificultad para estudiar son dependientes.

Establecimiento del nivel de significancia

$$
\alpha=0,05
$$

\section{Estadístico de prueba}

Tabla 9. Pruebas de chi-cuadrado 3

\begin{tabular}{|l|c|c|c|}
\hline & Valor & Gl & Valor $\mathrm{p}$ \\
\hline Chi-cuadrado de Pearson & 14,529 & 4 & 0,006 \\
Razón de verosimilitudes & 15,045 & 4 & 0,005 \\
N de casos válidos & 119 & & \\
\hline
\end{tabular}

Fuente: Investigación de campo

Elaboración: Autores

Cálculo del valor crítico y zona de rechazo

\begin{tabular}{|c|c|}
\hline $\mathrm{f}=$ & 3 \\
\hline $\mathrm{c}=$ & 3 \\
\hline $\mathrm{gl}=$ & 4 \\
\hline Valor crítico & $\mathbf{9 , 4 8 8}$ \\
\hline
\end{tabular}

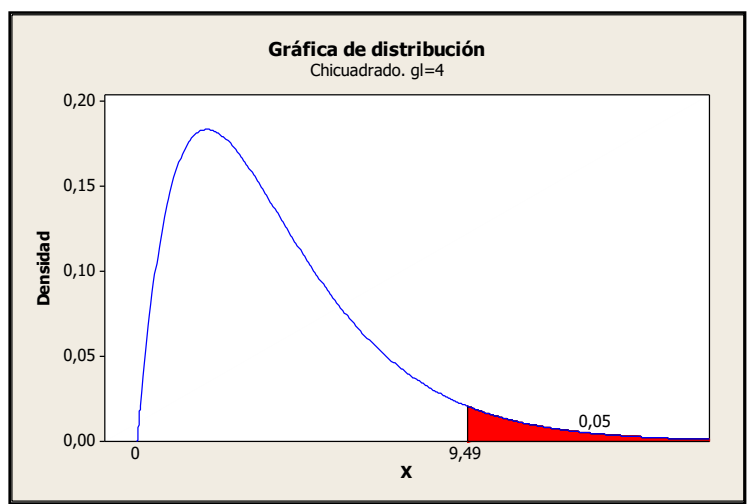

Gráfico No.3 Valor crítico y zona de rechazo 3 


\section{Regla de decisión}

Rechazar Ho si el estadístico de prueba es mayor que el valor crítico

No rechazar Ho si el estadístico de prueba es menor que el valor crítico

\section{Conclusión}

Como el estadístico de prueba es mayor que el valor crítico se procede a rechazar Ho, entonces, el deseo de retirarse de la carrera y la dificultad para estudiar son dependientes.

\section{Deserción y conformidad con la carrera}

\begin{tabular}{|c|c|c|c|c|}
\hline & & \multicolumn{2}{|c|}{ ¿Está conforme con su carrera? } & \multirow[b]{2}{*}{ Total } \\
\hline & & $\mathbf{S i}$ & No & \\
\hline \multirow{3}{*}{$\begin{array}{c}\text { ¿Quisiera o ha } \\
\text { querido } \\
\text { retirarse de la } \\
\text { carrera? }\end{array}$} & $\mathbf{S i}$ & 4 & 5 & 9 \\
\hline & No & 82 & 2 & 84 \\
\hline & A veces & 19 & 6 & 25 \\
\hline & Total & 105 & 13 & 118 \\
\hline
\end{tabular}

\section{Planteamiento de hipótesis}

Ho: El deseo de retirarse de la carrera y la conformidad con la misma son independientes.

H1: El deseo de retirarse de la carrera y la conformidad con la misma son dependientes.

\section{Establecimiento del nivel de significancia}

$$
\alpha=0,05
$$

\section{Estadístico de prueba}

Tabla 11. Pruebas de chi-cuadrado 4

\begin{tabular}{|l|c|c|c|}
\hline & Valor & Gl & Valor $\mathrm{p}$ \\
\hline Chi-cuadrado de Pearson & 28901 & 2 & $5,30 \mathrm{E}-07$ \\
Razón de verosimilitudes & 23,039 & 2 & $9,93 \mathrm{E}-06$ \\
N de casos válidos & 118 & & \\
\hline
\end{tabular}

Fuente: Investigación de campo

Elaboración: Autores 
Cálculo del valor crítico y zona de rechazo

\begin{tabular}{|c|c|}
\hline $\mathrm{f}=$ & 3 \\
\hline $\mathrm{c}=$ & 2 \\
\hline $\mathrm{gl}=$ & 2 \\
\hline Valor crítico & $\mathbf{5 , 9 9 1}$ \\
\hline
\end{tabular}

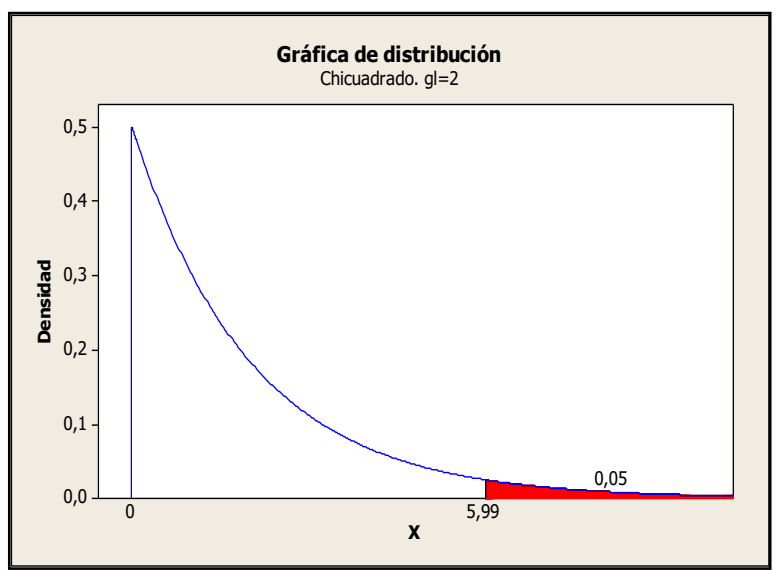

Gráfico No.4 Valor crítico y zona de rechazo 4

Fuente: Investigación de campo

Elaboración: Autores

\section{Regla de decisión}

Rechazar Ho si el estadístico de prueba es mayor que el valor crítico

No rechazar Ho si el estadístico de prueba es menor que el valor crítico

\section{Conclusión}

Como el estadístico de prueba es mayor que el valor crítico se procede a rechazar Ho, se concluye que el deseo de retirarse de la carrera y la conformidad con la misma son dependientes.

La Escuela Superior Politécnica de Chimborazo, recibe en sus diferentes facultades a estudiantes que provienen de varias ciudades del País y de cantones aledaños a Riobamba, así como de variados estratos económicos sociales; quienes en su gran mayoría vienen a residir en la ciudad de Riobamba y a vivir en condiciones muy diferentes a su vida de familia; una gran mayoría arrienda una modesta habitación y en muchos casos la comparten con otros estudiantes para ahorrar sus gastos de estadía. Estas condiciones de soledad y separación familiar -sumados a otros factores de tipo económico y de adaptación a la universidad y a su nueva forma de vida -, son en muchos casos la fuente de problemas personales psicosociales que los afectan e inciden en su rendimiento académico, y principalmente en su motivación. Lo cual se estima incide en su decisión de abandonar su carrera universitaria.

La deserción universitaria tiene un origen multicausal y las más recurrentes tienen que ver con los factores psicosociales que afectan a los estudiantes, el bajo rendimiento académico y las dificultades de financiar su carrera universitaria. 


\section{CONCLUSION}

Los estudiantes que participaron en esta investigación, evidencian la presencia de factores psicosociales que los afectan en el transcurso de su vida universitaria, así el desarraigo familiar incide en el estado emocional de los estudiantes universitarios, debido a que esto limita la interacción y comunicación familiar (11\% viven solos y el 54\% de los estudiantes provienen de otras provincias), el $60 \%$ de estudiantes no viven en familia. La mayoría, el $72 \%$ evidencia problemas personales que afectan ocasionalmente a su desempeño estudiantil; esto tiene que ver con los procesos psicológicos a su vez relacionados con la actitud y el comportamiento frente a la integración académica y social. La presencia ocasional de depresión y sentimientos de inseguridad (68\% a un $70 \%$ ) afecta su capacidad de integración social y su patrón de conducta adaptativa. Finalmente el tener un proyecto de vida no garantiza un adecuado nivel de motivación para estudiar; la muestra investigada refleja un $58 \%$ de motivación alta para continuar con la carrera universitaria.

Quienes no están conformes con la carrera universitaria, son los más propensos a plantearse abandonar sus estudios; en consecuencia, quienes están conformes con su carrera se adaptan con mayor facilidad al medio universitario y por tanto menos posibilidades de plantearse la deserción de su carrera. Los problemas de carácter psicosocial a nivel personal, sumados a factores del entorno tales como: inadaptación al medio universitario, problemas con profesores, abuso de alcohol entre otros, (42\%), son el segundo factor de incidencia en la intención de deserción; (después de los problemas académicos 49\%); al menos el $20 \%$ de los estudiantes no se adaptan con facilidad al medio universitario y se sienten afectados por el medio social, relacionado a nuevas costumbres, clima, horarios de clase, etc. Además, por la soledad al estar lejos de la familia y por la discriminación e inseguridad que sienten frente al medio universitario, constituyéndose así en un factor de deserción de la carrera universitaria. Por otra parte una orientación vocacional adecuada incide directamente, ya que el 98\% de los estudiantes que eligió la carrera por vocación, está conforme con la carrera y por tanto no tiene intenciones de desertar. No existen estudiantes que teniendo un rendimiento académico alto, quieran desertar; lo que denota, que el rendimiento académico es un factor muy importante en la intención de desertar. Mientras que los problemas de carácter económico que se presentan en el transcurso de la carrera inciden en un 9\%.en la intención de desertar de la carrera universitaria.

Los factores psicológicos, motivacionales son priorizados como los de mayor peso a la hora de considerar un apoyo institucional para ayudar a que los estudiantes eviten la deserción de la carrera, así el 59\% de estudiantes encuestados requirieron más un apoyo de tipo motivacional psicológico para evitar la deserción estudiantil, un 17\% consideró necesarios los incentivos 
tales como becas a los buenos estudiantes, el 19\% considera que se requiere de apoyo de tipo académico como tutorías extra clase, trabajos para recuperación de notas y el control del proceso académico de la carrera. El 39\% de estudiantes encuestados considera que la ESPOCH puede ayudar a evitar la deserción universitaria apoyando a sus estudiantes por medio de orientación psicológica, el 19\% considera que debe capacitarse a los docentes para que ofrezcan un mejor apoyo a los estudiantes y asumir también el rol de motivadores, además de 'propiciar una mejor vinculación estudiante-profesor con lo cual a la se evidencia que a la mirada de los estudiantes el docente debe tener un rol preponderante en apoyar a la estabilidad de los estudiantes en su carrera; además se plantea que la institución educativa debe propiciar la creación de grupos sociales para autoayuda para los estudiantes y apoyarlos por medio de tutorías extra clases y el otorgamiento de becas y el apoyo en emprendimientos y pasantías empresariales. El 75\% prioriza los aspectos de carácter psicosociales como la ayuda que requieren de sus propios compañeros para evitar la deserción, no se puede dejar de lado que igualmente requieren el apoyo en cuanto factores de orden académico.

\section{RECOMENDACIONES}

Se recomienda establecer un programa de control del desarrollo estudiantil a partir del ingreso a la carrera, que permita controlar tanto el desarrollo académico, integración universitaria, adaptación social y su desarrollo personal; de tal manera que, se convierta en un instrumento de gestión de la carrera universitaria que posibilite el control oportuno de los factores de deserción que afectan a los estudiantes, a partir del conocimiento de sus condiciones y características tanto socioeconómicas como personales. Esto significa detectar problemas a tiempo y canalizar oportunamente la ayuda para los estudiantes. A partir de contar con un sistema de información, se recomienda identificar los factores de riesgo de deserción de carácter psicosocial que afectan a los estudiantes, con la finalidad de establecer estrategias de retención o contención de los estudiantes en su carrera, articulados a cada una de las Facultades de la Escuela Superior Politécnica de Chimborazo

Definir estrategias que permitan acercar los servicios de bienestar estudiantil -hasta ahora centralizados en la administración central de la Escuela Superior Politécnica de Chimborazo - a las diferentes Facultades, de tal forma de facilitar la participación de cada Facultad y Escuela en la definición conjunta de un programa de apoyo estudiantil que facilite el acceso efectivo de estos servicios que por ley deben ser ofrecidos a los estudiantes universitarios y desarrollar un programa de apoyo para profesores con la finalidad de facilitar el proceso de guía y apoyo que deben ejercer los docentes en la interacción con sus estudiantes, para canalizar un adecuado apoyo 
respecto de problemas de carácter personal o familiar que afectan a los estudiantes universitarios, que permita atender a las dificultades que los estudiantes tienen para estudiar mediante la creación de grupos sociales de estudio para autoayuda, así como un programa permanente de tutorías extra clase en cada Facultad y Escuela.

Establecer dentro del departamento de bienestar estudiantil un programa de apoyo psicológico motivacional y de orientación sexual, para los estudiantes politécnicos, de manera descentralizada de tal forma que se pueda aplicar a nivel de Facultades y sus correspondientes Escuelas a fin de evitar factores que desencadenan en deserción universitaria.

\section{References:}

1. Álvarez, J. M. (1997). Etiología de un sueño o el abandono de la universidad por parte de los estudiantes por factores no académicos. Bogotá: Universidad Autónoma de Colombia

2. BREA de Cabra, M. (2005). Deserción en la educación superior pública en República Dominicana. Psicologíacientifica.com. Recuperado el 10 de mayo, 2006 de http://www.psicologiacientifica.com.

3. CORTEZ Baldiviezo Eduardo. (2005). Repitencia y deserción universitaria en América Latina. IES/2005/ED/PI/1.

4. DÍAZ - PERALTA, C. (2008). Modelo conceptual para la deserción estudiantil universitaria chilena. Estudios Pedagógicos, 34(2), 65-86. Donoso,S.,

5. DIAZ, (2009), Modelo conceptual para la deserción estudiantil universitaria chilena Universidad Católica de la Santísima Concepción, Código Postal: 409.

6. Giovagnoli, P. (2002). Determinantes de la deserción y graduación universitaria: una aplicación utilizando modelos de duración. Universidad Nacional de la Plata, Argentina.

7. GONZÁLEZ, L. (2005). Estudio sobre la repitencia y deserción en la educación superior chilena. IES/2005/ED/PI/45.

8. MONTERO, M. (2000). Elección de carrera profesional: visiones, promesas y desafíos. México: Universidad Autónoma de Ciudad de Juárez.

9. SELAME, T. (1994). Antecedentes y reflexiones sobre la deserción estudiantil en la universidad de Santiago de Chile. Valdivia: USC

10. SPADY, W. G. (1970), "Dropouts from higher education: An interdisciplinary review and synthesis"; en Interchange, 1(1),

11. TINTO, Vincent (1982) Limits of Theory and Practice in Student Attrition. The Journal of Higher Education, Vol. 53, $\mathrm{N}^{\circ}$ 6, pp: 687-700 (Nov. - Dec. 1982) 